\title{
Energy-efficient Off-Body Data Transmission by Means of Antenna Diversity and Tracking of the Time-Variant Wireless Channel State
}

\author{
Patrick Van Torre*‡, Luigi Vallozzi*, Hendrik Rogier*, Marc Moeneclaey ${ }^{\dagger}$, Jo Verhaevert ${ }^{\ddagger}$ \\ *INTEC, Ghent University \\ $\dagger^{\text {TELIN, Ghent University }}$ \\ Sint-Pietersnieuwstraat 41, 9000 Ghent, Belgium \\ Patrick.VanTorre@UGent.be \\ ‡INWE, University College Ghent \\ Valentin Vaerwyckweg 1, 9000 Ghent, Belgium
}

\begin{abstract}
Textile antennas integrated into the garment of rescue workers offer a convenient way for implementing an offbody communication system. When operating indoors, the link reliability is compromised by fading and shadowing. For a SISO system, the transmitted power needs to be very large to allow an acceptable bit error rate at the receiver. Employing front and back dual-polarized textile antennas creates fourth order diversity at the body. Equipping also the base-station with such a pair of antennas results in diversity at both sides, in this case realizing a $4 \times 4$ MIMO system. The MIMO techniques counter the effects of fading and shadowing and allow the system to achieve low bit error rates at the receiver with a minimum of transmitted power. Long data frames are used with a minimum pilot symbol overhead, also reducing the transmitted power per data bit. Although the propagation channel is time-varying during the transmitted frame, the channel variation is tracked at the receiver with a minimal processing power.
\end{abstract}

\section{INTRODUCTION}

Wearable textile systems aim at improving safety of rescue workers by allowing real-time monitoring of their activity and life signs. In such systems wearable RFIDs, sensors and especially antennas are usually employed in order to implement data communication and monitoring, for example by means of digital video streaming.

In these applications power efficiency is a very important matter. It is desirable for a wearable system to have a very low power consumption, especially for autonomous systems for which the necessary energy for the operation of the devices is scavenged from the surrounding environment and ideally no additional power supply is present. In particular, the hardware and software that realizes wireless communication between the system and the external world (i.e. wearable antennas, transceiver and signal processing) must be designed in such a way to ensure a required value of Bit Error Rate (BER) using the smallest possible amount of transmit power.

Unfortunately, several adversities must be overcome in order to realize power efficient wearable communication systems. The wireless channel in which wearable textile systems are employed, often represents a hostile environment, making the realization a of reliable and power-efficient wireless communication system a challenging task. In fact, for rescue-worker applications in particular, transmitter and receiver are typically in relative motion inside a rich-scattering environment, giving rise to phenomena that are detrimental for power efficiency, such as fading and shadowing. The combination of these two phenomena produces a random fluctuation of the received signal, with deep fades that produce errors in the received data stream. In such cases, to ensure a given value of bit error rate, it is necessary to increase the transmit power with respect to the ideal case without fading and shadowing.

In order to combat fading and shadowing and to be able to use a lower transmit power for a given BER (realizing thus a power-efficient system) we propose a wearable communication system with the following characteristics:

1) Multiple wearable antennas integrated at different locations of the clothing worn on a human body, implementing receive diversity. Multiple antenna reception with combining schemes is an effective method to combat channel variability: placing two dual-polarized antennas at the front and back of the human body avoids shadowing by this body, and combining the four received signals relying on an appropriate scheme (i.e. MRC) allows to minimize signal fading. Such diversity scheme requires the channel knowledge at receive side, which can be obtained by estimation.

2) As a second feature for improving power efficiency of the system, we propose robust channel tracking at the receive side. In time-variant channels, data transmission is often performed by sending short symbol packets, whose duration is smaller than the channel coherence time (i.e. the time interval during which the channel can be considered constant). Each packet is composed of a pilot symbol sequence for channel estimation (i.e. the packet overhead), followed by a data symbol field (i.e. the actual information to transmit). The use of many short packets does not represent a power efficient 
solution, since it implies the use of a large amount of transmit power for the overhead, which does not contain useful information, but is required for channel estimation only. For this reason, we propose the use of longer packets (with a duration larger than the channel coherence time) combined with dynamic channel estimation or channel tracking, resulting in a reduced overhead with consequent decrease of the required transmit power. The channel tracking is performed by means of a simple algorithm, causing no significant extra power demands for the receiving system.

The system was realized and its performance was investigated by means of a measurement campaign conducted in an indoor office environment. A test-person was equipped with the system and, by means of a measurement test-bed, a real data transmission was implemented with the test person moving around in the considered environment. Results, in particular BER curves, showed that antenna diversity in combination with channel tracking allows to save a large amount of transmit power (for a given required BER) and represent thus a valid scheme for power-efficient communication systems.

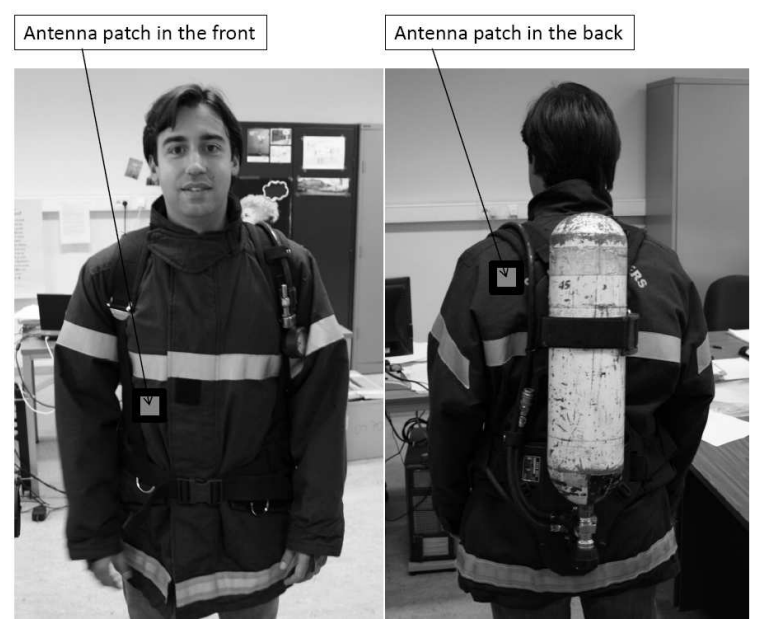

Fig. 1. Locations of the dual-polarized patch antennas integrated in the fire-fighter's garment.

\section{Measurement Setup}

\section{A. Wearable antenna system}

The prototyped wearable antenna system represents the receive side of the communication link, integrated on a firefighter jacket worn by a real human subject. Such a system is composed of two dual-polarized textile patch antennas [1], whose geometrical scheme is shown in Fig.2. This antenna is capable of simultaneously receiving (or transmitting) two independent nearly-orthogonal signals, enabling the implementation of $2^{\text {nd }}$-order diversity with a single antenna. In the proposed antenna system two such antennas where used, the first placed at the front of the human torso and the second at its back side, resulting in a total diversity of $4^{\text {th }}$ order. The type of receive diversity realized by the proposed scheme can be seen as a combination of polarization diversity, provided singularly by each wearable antenna, and front-toback diversity, provided by the opposite orientation of the two wearable antennas. Such a kind of diversity was already proven to be effective against the effects of fading and shadowing on the quality (in terms of bit error rate) of an indoor off-body wireless communication between a fixed base station and a moving subject [2], [3].

The transmit side of the communication link is also composed of two dual-polarized patch antennas, placed at fixed positions, differing in height and location, inside the indoor environment, in order to ensure sufficient spatial decorrelation between signals transmitted by different antennas.

The wireless communication between the TX and RX antenna systems is realized by a wireless testbed, composed of a TX and a RX unit, to which the terminals of the TX and RX antenna systems are connected respectively. The testbed units are controlled via Matlab by means of PCs.

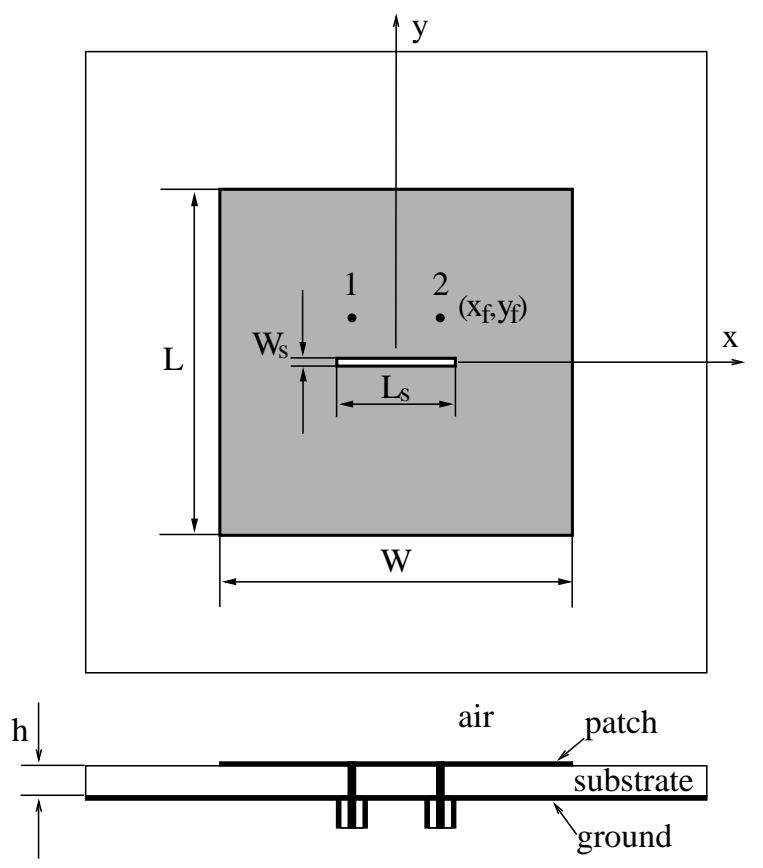

Fig. 2. Geometrical description of the dual-polarized textile patch antenna.

\section{B. Transmitted frame structure}

Due to memory restrictions at the transmitter, short frames are transmitted repetitively. These frames include 300 pilot symbols transmitted in separate time-slots for each transmit channel as well as 528 symbols of space-time coded data transmitted on all four transmit channels. In the post-processing stage we can choose whether to use the pilot information or not.

The structure of the transmitted frame is shown in Table I. The total duration of the frame is nearly $2 \mathrm{~ms}$, followed by a $128 \mu s$ transmit gap. The symbol rate is $10^{6}$ QPSK symbols per second and the transmit frequency is $2.45 \mathrm{GHz}$. 


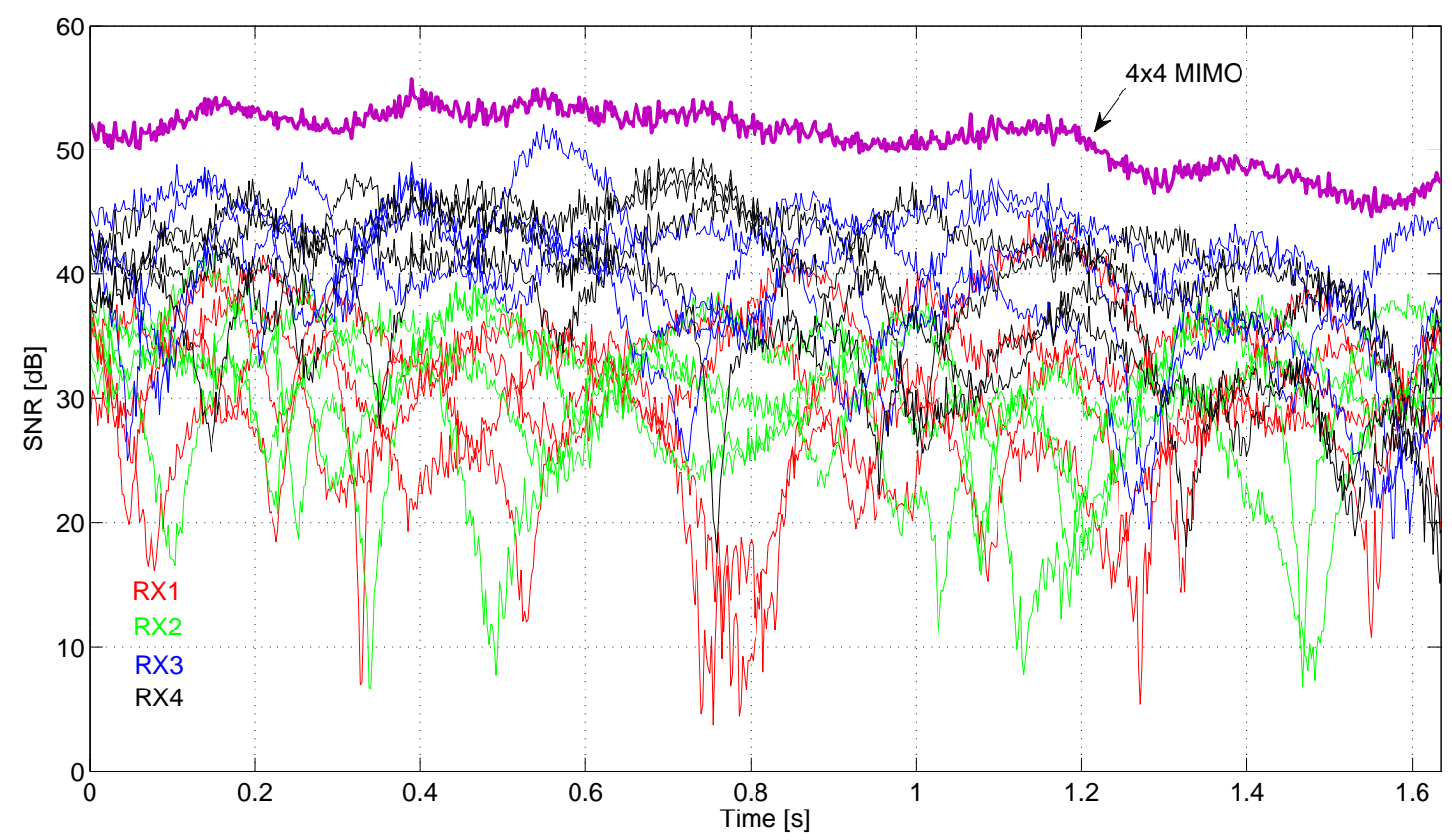

Fig. 3. SNR for the walking firefighter as a function of time for the 16 SISO channels and for the $4 \times 4$ MIMO channel composed by them.
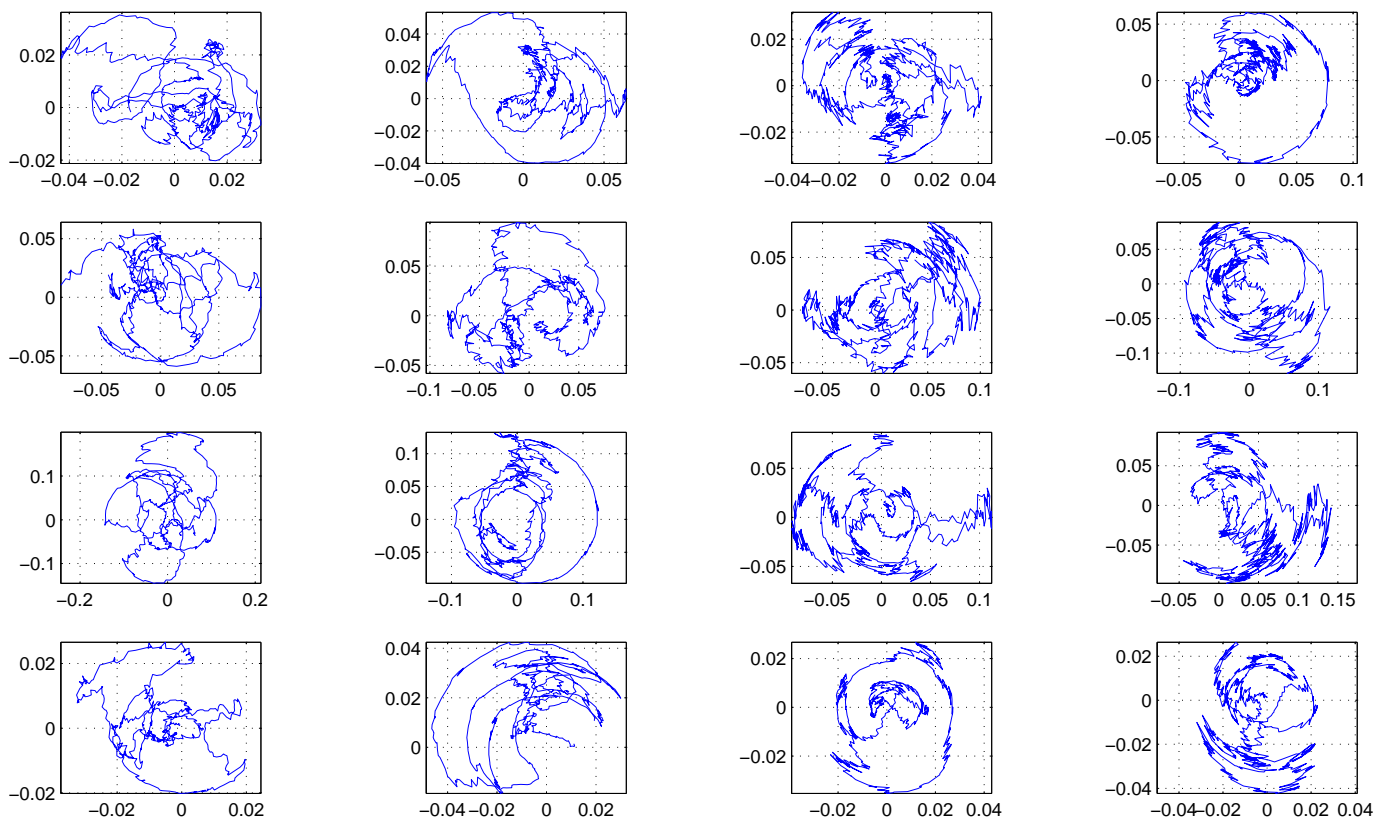

Fig. 4. Complex channel estimates for the 16 SISO channels composing the $4 \times 4$ MIMO channel, for the walking firefighter in NLoS, during 1.6 seconds. 
TABLE I

STRUCTURE OF THE TRANSMITTED SIGNALS, INDICATING TIMING OF (P)ILOT AND (D)ATA TRANSMISSION.

\begin{tabular}{|c|c|c|c|c|}
\hline TX channel & \multicolumn{4}{|c|}{ Timeslot } \\
\hline TX1 & $\mathrm{P}$ & & D & \\
\hline TX2 & & $\mathrm{P}$ & D & \\
\hline TX3 & & & D & $\mathrm{P}$ \\
\hline TX4 & & & D & \\
\hline
\end{tabular}

The receiver samples the signal synchronously on its four input channels until the receiver memory is full, then containing MIMO channel samples for a duration of 1.66 seconds. The repetitive frame structure allows the demodulation and detection of nearly the full recorded dataset. Without repetition a large part of the recorded data would be unusable due to the missing pilot symbols for initial channel estimation. Whether the further pilot symbols are used or not can be chosen in the demodulation process, thus allowing a performance comparison of channel tracking with or without further pilot assistance.

\section{Measurement Results}

\section{A. Time-domain behavior}

1) Evolution of the SNR: Fig. 3 illustrates the performance of the system for one transmitted burst of 1.6 seconds duration. Signals for different receiving channels are displayed in different colors. Four curves are shown for each receiving channel, corresponding to the four transmitted signals. In total 16 SISO channels are present, each experiencing an enormous variation in signal-to-noise ratio (SNR). The average bit error rate experienced by a system is predominantly determined by the minima in the SNR curve, producing the largest share of bit errors. Hence the transmitted power has to be dramatically increased in order to still achieve an acceptable bit error rate during the signal minima.

The average SNR at the detector is $12.0 \mathrm{~dB}$ higher for the MIMO system, compared to the average SISO channel. This corresponds to two times $6 \mathrm{~dB}$ array gain from the 4antenna arrays at the transmitter and the receiver. When the total transmitted power for the MIMO system is equal to the transmitted power for a SISO system, no transmit array gain is present. In this case, $6 \mathrm{~dB}$ less performance increase is obtained.

The minimum SNR at the detector is $40.9 \mathrm{~dB}$ higher for the MIMO system, compared to the minimum for the worst SISO channel. This has a very important effect on the average bit error rate.

Exploiting MIMO techniques to counter the fading, as well as taking advantage of the antenna array gain at both sides of the link results in the top curve, labeled $4 \times 4$ MIMO. From the graph, two advantages of the MIMO system are clear:

- The average SNR is significantly higher for the MIMO system.

- The SNR variation is drastically reduced by the MIMO system.
2) Evolution of the complex channel estimates: Employing MIMO techniques requires accurate channel estimates to perform proper space-time decoding and demodulation. The amplitude as well as the phase of these channel estimates fluctuate dramatically over a period of 1.6 seconds, as illustrated by the complex channel estimates for the 16 SISO channels composing the MIMO channel in Fig. 4.

A correct demodulation will depend on accurate channel estimates, tracking the channel variation during the transmitted frame. For this purpose extra pilot symbols can be transmitted, at regular intervals between the data symbols. We will present results with and without these extra pilot symbols. The timevarying channel can be tracked without significant processing overhead at the receiver, as illustrated in [4], if a small residual channel estimation error can be tolerated. Channel tracking schemes, as documented in [5]-[10] can track the channel more accurately at the price of a higher computational complexity.

\section{B. Bit error characteristics}

Fig. 5 displays bit error curves calculated for the 16 SISO channels and for the $4 \times 4$ MIMO channel composed by them. For QPSK modulation these curves are calculated based on the recorded set of amplitudes for the pilot symbols as outlined in [11]. As a comparison, theoretical curves for uncorrelated equal gain Rayleigh fading channels are also displayed. Practical demodulation requires either the use of pilot symbols or dynamic channel tracking to follow the dramatic channel variation shown in Figs. 3 and 4. Characteristics are displayed for both channel estimation systems.

- The lower Rayleigh curve is for theoretical Rayleigh fading with 16-th order diversity, calculated with the array gain for a $4 \times 4$ MIMO system. This optimal curve requires 16 uncorrelated Rayleigh fading channels with equal average channel gains.

- The characteristic labeled Perfect CSI (Channel State Information) represents the performance with perfect channel estimation. This curve is calculated based on the recorded set of signal-to-noise ratios for the 16 SISO channels. The performance is limited by channel correlation and unequal average channel gains, resulting in a $1.5 \mathrm{~dB}$ worse performance than the theoretical optimum, compared at $\mathrm{BER}=2 \cdot 10^{-4}$.

- The curve labeled Pilot CSI is found by counting bit errors after full receiver processing, including channel estimation errors occurring due to noisy pilot symbols and continuous channel variation, influencing the performance of demodulation and detection. The channel estimation errors reduce the performance by $2.4 \mathrm{~dB}$ at $\mathrm{BER}=2 \cdot 10^{-4}$, compared to perfect CSI. Note that although, the average $E_{b} / N_{0}$ per input antenna is $7 \mathrm{~dB}$, the pilot symbols for estimation of the individual SISO channels experience Rayleigh fading without diversity and hence can sometimes be very noisy.

- The characteristic labeled Data CSI is found in a similar way, but now the continuous channel estimation is 
realized without using the pilot symbols and employing only data driven feedback. The performance is reduced by less than $0.3 \mathrm{~dB}$, measured at $E_{b} / N_{0}=7 \mathrm{~dB}$ and $\mathrm{BER}=2 \cdot 10^{-4}$. The performance degradation is caused by filter delay and decision errors. For lower $E_{b} / N_{0}$ values, decision errors occur more frequently resulting in a further reduced performance. For $E_{b} / N_{0}=6 \mathrm{~dB}$, BER $>10^{-2}$ whereas in case of pilot-based CSI, $\mathrm{BER}=5 \cdot 10^{-4}$.

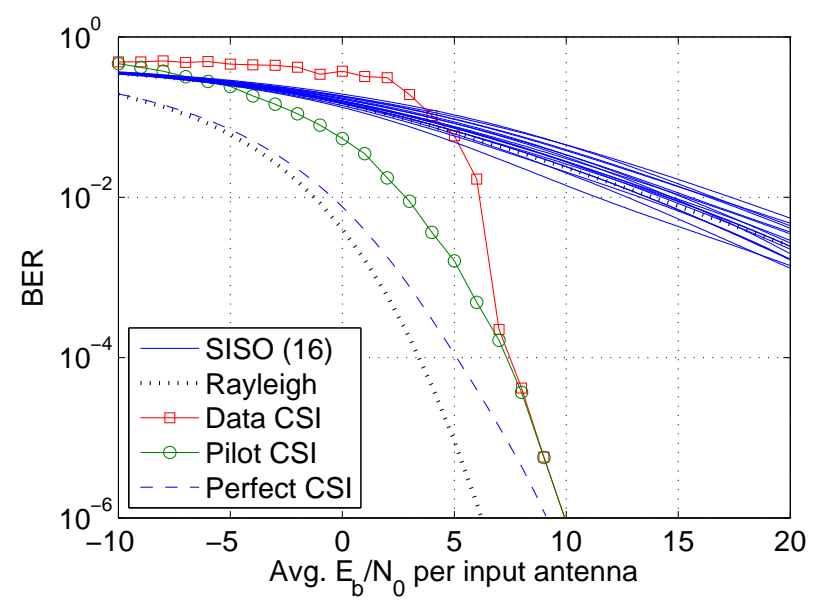

Fig. 5. Bit error characteristics for QPSK modulation on the 16 SISO channels compared to theoretical Rayleigh fading curve. Bit error characteristics for the $4 \times 4$ MIMO channel, as calculated based on perfect channel estimation, on pilot symbols and on data driven channel tracking.

The $4 \times 4$ MIMO system with data driven channel tracking achieves a good performance for bit error rates lower than $10^{-4}$. The channel tracking employs decision oriented feedback on the space-time decoded signal after maximal ratio combining at the receiver, performing much more reliably than achievable in a SISO system thanks to the drastically reduced signal variation at this point in the processing.

The measured error performance of $\mathrm{BER}=2 \cdot 10^{-4}$ at $E_{b} / N_{0}=7 \mathrm{~dB}$, requires an $E_{b} / N_{0}=31 \mathrm{~dB}$ for theoretical Rayleigh fading channels without diversity. Note that, even for this high $E_{b} / N_{0}$, the latter performance is only obtained with perfect channel estimation.

The MIMO system achieves this performance in realistic conditions at a received $E_{b} / N_{0}$ per input antenna that is $24 \mathrm{~dB}$ lower than required for the theoretical optimal SISO performance for a Rayleigh fading channel. Pilot symbols are only used during 1.2 milliseconds of the 1.6 second received frame, hence the overhead introduced by these pilots is insignificant. Therefore, for the $4 \times 4$ MIMO system with data driven decision oriented feedback, the total transmitted power can be reduced by $24 \mathrm{~dB}$ compared to the required power for a SISO link.

\section{CONCLUSIONS}

For indoor non line-of-sight wireless off-body communication at $2.45 \mathrm{GHz}$, MIMO techniques allow us the counter the channel variation and hence comparable bit error rates are achieved at a much lower total transmit power. Dynamic channel tracking is possible with data driven decision oriented feedback, reducing the pilot symbol overhead for the complete transmission to an insignificant proportion.

The residual channel estimation error that is inherently present when using dynamic channel tracking causes only a limited performance degradation for bit error rates of practical use. Bit error characteristics calculated for the measured channels illustrate this behavior, achieving a BER $=2 \cdot 10^{-4}$ at $24 \mathrm{~dB}$ less total transmitted power. Reducing the transmitted power over more than two orders of magnitude results in significant energy savings and a longer autonomy for a battery operated or energy-scavenging communication system.

\section{ACKNOWLEDGMENTS}

This work was supported by the Fund for Scientific Research - Flanders (FWO-V) by Project "Advanced spacetime processing techniques for communication through multiantenna systems in realistic mobile channels.".

\section{REFERENCES}

[1] L. Vallozzi, H. Rogier, and C. Hertleer, "Dual polarized textile patch antenna for integration into protective garments," Antennas and Wireless Propagation Letters, IEEE, vol. 7, pp. 440 -443, 2008.

[2] L. Vallozzi, P. Van Torre, C. Hertleer, H. Rogier, M. Moeneclaey, and J. Verhaevert, "Wireless communication for firefighters using dualpolarized textile antennas integrated in their garment," Antennas and Propagation, IEEE Transactions on, vol. 58, no. 4, pp. $1357-1368$, April 2010.

[3] P. Van Torre, L. Vallozzi, L. Jacobs, H. Rogier, M. Moeneclaey, and J. Verhaevert, "Characterization of measured indoor off-body MIMO channels with correlated fading, correlated shadowing and constant path loss," Wireless Communications, IEEE Transactions on, vol. PP, no. 99, pp. $1-10,2011$.

[4] P. Van Torre, L. Vallozzi, H. Rogier, M. Moeneclaey, and J. Verhaevert, "Channel characterization and robust tracking for diversity reception over time-variant off-body wireless communication channels," EURASIP J. Adv. Signal Process, vol. 2010, pp. 7:1-7:13, February 2010. [Online] Available: http://dx.doi.org/10.1155/2010/978085

[5] C. Komninakis, C. Fragouli, A. Sayed, and R. Wesel, "Multi-input multi-output fading channel tracking and equalization using kalman estimation," Signal Processing, IEEE Transactions on, vol. 50, no. 5, pp. $1065-1076$, May 2002.

[6] Y. Yapici and A. O. Yilmaz, "Low-complexity iterative channel estimation and tracking for time-varying multi-antenna systems," in Personal, Indoor and Mobile Radio Communications, 2009 IEEE 20th International Symposium on, Sept. 2009, pp. $1317-1321$.

[7] A. Wolfgang, J. Akhtman, S. Chen, and L. Hanzo, "Reduced-complexity near-maximum-likelihood detection for decision feedback assisted space-time equalization," Wireless Communications, IEEE Transactions on, vol. 6, no. 7, pp. 2407 -2411, July 2007.

[8] B. Balakumar, S. Shahbazpanahi, and T. Kirubarajan, "Joint mimo channel tracking and symbol decoding using kalman filtering," Signal Processing, IEEE Transactions on, vol. 55, no. 12, pp. 5873 -5879, Dec. 2007.

[9] K. Huber and S. Haykin, "Improved bayesian MIMO channel tracking for wireless communications: incorporating a dynamical model," Wireless Communications, IEEE Transactions on, vol. 5, no. 9, pp. 2458 -2466 , Sept. 2006.

[10] J. Choi, H. Yu, and Y. Lee, "Adaptive MIMO decision feedback equalization for receivers with time-varying channels," Signal Processing, IEEE Transactions on, vol. 53, no. 11, pp. 4295 - 4303, Nov. 2005.

[11] P. Van Torre, L. Vallozzi, C. Hertleer, H. Rogier, M. Moeneclaey, and J. Verhaevert, "Indoor off-body wireless MIMO communication with dual polarized textile antennas," Antennas and Propagation, IEEE Transactions on, vol. 59, no. 2, pp. 631 -642, Feb. 2011. 